\title{
Erratum to: Spongiimicrobium salis gen. nov., sp. nov., a bacterium of the family Flavobacteriaceae isolated from a marine sponge
}

\author{
Jaewoo Yoon $^{1} \cdot$ Kyoko Adachi $^{2,4} \cdot$ Hiroaki Kasai $^{3}$
}

Published online: 16 July 2016

(C) Springer-Verlag Berlin Heidelberg 2016

\section{Erratum to: Arch Microbiol DOI 10.1007/s00203-016-1227-3}

Subsequent to publication it has been noticed that the polar lipid profile given in the above paper is incorrect. The composition of the polar lipid profile has been corrected in the respective sections of the paper as shown below.

\begin{abstract}
A Gram-stain-negative, strictly aerobic, paleyellow pigmented, rod-shaped, chemoheterotrophic bacterium, designated $\mathrm{A} 6 \mathrm{~F}-11^{\mathrm{T}}$, was isolated from a marine sponge collected in Japan. Phylogenetic analysis based on the 16S rRNA gene sequence indicated that the novel marine strain was affiliated with the family Flavobacteriaceae of the phylum Bacteroidetes and that it shared the highest $(92.9 \%)$ sequence similarity with Arenibacter palladensis LMG $21972^{\mathrm{T}}$. The strain could be differentiated
\end{abstract}

The online version of the original article can be found under doi:10.1007/s00203-016-1227-3.

Jaewoo Yoon

jwyoon@kmu.ac.kr

1 College of Pharmacy, Keimyung University, 1095

Dalgubeoldaero, Dalseo-Gu, Daegu 42601,

Republic of Korea

2 Marine Biotechnology Institute, 3-75-1 Heita, Kamaishi, Iwate 026-0001, Japan

3 Marine Biosciences Kamaishi Research Laboratory, Kitasato University, 160-4, Utou, Okirai, Sanriku-cho, Ofunato, Iwate 022-0101, Japan

4 Basic Research Laboratories, Nippon Steel \& Sumikin Chemical Co., Ltd., 1-Tsukiji, Kisarazu, Chiba 292-0835, Japan phenotypically from related members of the family Flavobacteriaceae. The major fatty acids of strain A6F-11 ${ }^{\mathrm{T}}$ were iso-C15:1 G, iso-C15:0, C16:1 $\omega 6 \mathrm{c}$ and/or C16:1 $\omega 7 \mathrm{c}$ and iso-C17:0 3-OH. A polar lipid profile consisted of phosphatidylethanolamine, two unidentified aminolipids and two unidentified lipids. The DNA G+C content was $34.7 \mathrm{~mol} \%$, and the major respiratory quinone was menaquinone 6 (MK-6). From the distinct phylogenetic position and combination of genotypic and phenotypic characteristics, the strain is considered to represent a novel taxon in the family Flavobacteriaceae, for which the name Spongiimicrobium salis gen. nov., sp. nov. is proposed. The type strain of $S$. salis gen. nov., sp. nov. is A6F-11 ${ }^{\mathrm{T}}$ $\left(=\right.$ KCTC $42753^{\mathrm{T}}=$ NBRC $\left.111401^{\mathrm{T}}\right)$.

\section{Results and discussion}

\section{Chemotaxonomic characteristics}

The polar lipids of strain $\mathrm{A} 6 \mathrm{~F}-11^{\mathrm{T}}$ were determined to be composed of phosphatidylethanolamine, two unidentified aminolipids and two unidentified lipids (Supplementary Fig. 1).

\section{Description of Spongiimicrobium salis sp. nov}

The major polar lipids are phosphatidylethanolamine, two unidentified aminolipids and two unidentified lipids (Table 1). 
Table 1 Differential characteristics of strain A6F-11 ${ }^{\mathrm{T}}$ and other closely related taxa

\begin{tabular}{|c|c|c|c|c|c|c|}
\hline Characteristic & 1 & 2 & 3 & 4 & 5 & 6 \\
\hline Pigmentation & Pale-yellow & Orange to dark-orange & Dark-orange & Yellow to dark-orange & Yellow & Dark-orange \\
\hline Gliding motility & - & $\pm\left(-{ }^{a}\right)$ & + & $\pm\left(+^{b}\right)$ & + & + \\
\hline $\begin{array}{l}\text { Temperature range for } \\
\text { growth }\left({ }^{\circ} \mathrm{C}\right)\end{array}$ & $15-37$ & $4-40$ & $4-37$ & $4-50$ & $4-40$ & $4-50$ \\
\hline $\mathrm{pH}$ range for growth & $6-8$ & $6-9.6$ & ND & $5.5-10.5$ & $5-9$ & $6-8.5$ \\
\hline $\begin{array}{l}\text { Highest } \mathrm{NaCl} \text { tolerance } \\
(\%, \mathrm{w} / \mathrm{v})\end{array}$ & 5 & 8 & 6 & 10 & 10 & 10 \\
\hline Nitrate reduction & - & $\pm\left(+^{a}\right)$ & - & $\pm\left(+^{b}\right)$ & $\pm\left(-{ }^{\mathrm{d}}\right)$ & + \\
\hline \multicolumn{7}{|l|}{ Hydrolysis of } \\
\hline Gelatin & + & $\pm\left(-{ }^{a}\right)$ & - & $\pm\left(-^{b}\right)$ & + & + \\
\hline ONPG & + & + & + & $\pm\left(-^{\mathrm{b}}\right)$ & + & + \\
\hline Starch & - & - & - & $\pm\left(+^{b}\right)$ & + & $\pm\left(+^{\mathrm{e}}\right)$ \\
\hline Urea & - & $\pm\left(+^{\mathrm{a}}\right)$ & - & $\pm\left(-^{c}\right)$ & - & - \\
\hline \multicolumn{7}{|l|}{ Acid production from } \\
\hline Cellobiose & + & + & + & $\pm\left(-^{b}\right)$ & + & $\pm\left(+^{e}\right)$ \\
\hline Galactose & + & $\pm\left(+^{\mathrm{a}}\right)$ & + & $\pm\left(-^{b}\right)$ & + & $\pm\left(-^{e}\right)$ \\
\hline Glucose & - & + & + & $\pm\left(-^{b}\right)$ & + & $\pm\left(-^{e}\right)$ \\
\hline Maltose & - & $\pm\left(+^{\mathrm{a}}\right)$ & + & $\pm\left(-^{b}\right)$ & $\pm\left(+^{\mathrm{d}}\right)$ & $\pm\left(+^{\mathrm{e}}\right)$ \\
\hline Melibiose & + & $\pm\left(+^{\mathrm{a}}\right)$ & + & $\pm\left(-^{\mathrm{b}}\right)$ & + & - \\
\hline Lactose & - & + & + & $\pm\left(-^{b}\right)$ & + & - \\
\hline Raffinose & - & $\pm\left(+^{\mathrm{a}}\right)$ & + & $\pm\left(-^{\mathrm{b}}\right)$ & $\pm\left(-{ }^{\mathrm{d}}\right)$ & $\pm\left(-^{e}\right)$ \\
\hline Polar lipids & PE, 2UAL, 2UL & PE, 2UL & ND & PE, 2UAL, 4UL & 2UL, UAL, UAPL & ND \\
\hline DNA G+C content $(\mathrm{mol} \%)$ & 34.7 & $37-40$ & $35.8-41$ & $34.2-41.1$ & $31.6-35.4$ & $36.1-43.4$ \\
\hline
\end{tabular}

Strains: 1 A6F-11 ${ }^{\mathrm{T}}$ (Spongiimicrobium salis gen. nov., sp. nov.; present study), 2 Arenibacter spp. (Ivanova et al. 2001; Nedashkovskaya et al. 2006; Sun et al. 2010; Jeong et al. 2013), 3 Kriegella aquimaris KMM $3665^{\mathrm{T}}$ (Nedashkovskaya et al. 2008), 4 Maribacter spp. (Nedashkovskaya et al. 2004a; Zhang et al. 2009; Hu et al. 2015; Jackson et al. 2015; Park et al. 2015), 5 Cellulophaga spp. (Kahng et al. 2009; Park et al. 2012), 6 Zobellia spp. (Barbeyron et al. 2001; Nedashkovskaya et al. 2004b)

Data for the type strain of the type species (A. latericius KMM $426^{\mathrm{T}}$, M. sedimenticola KMM $3903^{\mathrm{T}}$, C. lytica ATCC $23178^{\mathrm{T}}$ and Z. galactanovora Dsij $^{\mathrm{T}}$ ) from a Jeong et al. 2013; b Nedashkovskaya et al. 2004a; c Park et al. 2015; d Park et al. 2012; e Nedashkovskaya et al. 2004b

$P E$ phosphatidylethanolamine, UAL unidentified aminolipid, $U L$ unidentified lipid, UAPL unidentified aminophospholipid, + positive, - negative, $N D$ no data 